\title{
纳米铌酸钾钠陶瓷的制备及性能
}

\author{
王超 ${ }^{1}$, 陈 静 $^{1}$, 陈红丽 ${ }^{1}$, 侯育冬 ${ }^{2}$, 朱满康 ${ }^{2}$ \\ (1. 北京物资学院 物流学院, 北京 $101149 ; 2$. 北京工业大学 新型功能材料教育部重点实验室, 北京 100124)
}

摘 要: 以新型溶胶一凝胶法制备的平均晶粒尺寸为 $30 \mathrm{~nm}$ 的铌酸钾钠粉体为原料, 采用放电等离子体烧结工艺, 在 烧结温度为 $900^{\circ} \mathrm{C}$, 压力 $30 \mathrm{MPa}$, 烧结时间 $1 \mathrm{~min}$ 的条件下, 制备得到纯正交相, 相对密度高达 $99 \%$ 以, 平均晶 粒尺寸为 $40 \mathrm{~nm}$ 的纳米铌酸钾钠陶瓷, 并对该陶瓷的相结构、微观形貌、介电性能和铁电性能进行了研究。结果表 明, 与普通微米晶陶瓷不同, 纳米铌酸钾钠陶瓷的室温介电常数仅为 341 , 并且随温度变化不明显, 表现出明显的 介电弛豫现象, 弥散因子 $\gamma$ 为 1.60 , 并具有明显的电滞回线, 矫顽场强度为 $13.5 \mathrm{kV} / \mathrm{cm}$, 剩余极化为 $1.5 \mu \mathrm{C} / \mathrm{cm}^{2}$ 。尺 寸降低所引起的纳米铌酸钾钠陶瓷中晶界相所占的比例增大是其性能变化的主要原因, 并且可以推断, 如果铌酸 钾钠陶瓷具有 “临界尺寸”，那么其值应该在 $40 \mathrm{~nm}$ 以下。

关 键 词: 铌酸钾钠; 纳米陶瓷; 介电弛豫; 电滞回线

中图分类号: TM282 文献标识码: A

\section{Preparation and Properties of Nanocrystalline Potassium Sodium Niobate Ceramics}

\author{
WANG Chao ${ }^{1}$, CHEN Jing ${ }^{1}$, CHEN Hong-Li ${ }^{1}$, HOU Yu-Dong ${ }^{2}$, ZHU Man-Kang ${ }^{2}$
}

(1. Logistics School, Beijing Wuzi University, Beijing 101149, China; 2. Key Laboratory of Advanced Functional Materials of China Education Ministry, Beijing University of Technology, Beijing 100124, China)

\begin{abstract}
Using average $30 \mathrm{~nm}$ grain sized potassium sodium niobate powders obtained by a novel Sol-Gel method as starting materials, nanocrystalline potassium sodium niobate ceramics with pure orthorhombic phase, relative density of above $99 \%$ and grain size of $40 \mathrm{~nm}$ were prepared by spark plasma sintering method under sintering temperature of $900{ }^{\circ} \mathrm{C}$, sintering pressure of $30 \mathrm{MPa}$ and sintering time of $1 \mathrm{~min}$. The phase structure, micro-morphology, dielectric properties and ferroelectric properties of the ceramics were investigated. The results show that, differently from common micro-grain ceramics, dielectric constant of nanocrystalline potassium sodium niobate ceramics decreases to 341 , which changes little with the temperature, and the ceramics have obvious dielectric relaxation (dispersion factor $\gamma$ is 1.60), and a well saturated ferroelectric hysteresis loop with coercive field of $13.5 \mathrm{kV} / \mathrm{cm}$ and remanent polarization of $1.5 \mu \mathrm{C} / \mathrm{cm}^{2}$. The abnormal properties can be attributed to the significant increasing content of grain boundary in nanocrystalline potassium sodium niobate ceramics, and it can be expected that if potassium sodium niobate ceramic has the critical size at room temperature, it would be less than $40 \mathrm{~nm}$.
\end{abstract}

Key words: potassium sodium niobate; nanocrystalline ceramic; dielectric relaxation; ferroelectric hysteresis loop 
目前, 商用的压电陶瓷仍以 $\mathrm{Pb}(\mathrm{Zr}, \mathrm{Ti}) \mathrm{O}_{3}$ (锆铁酸 铅, 缩写为 PZT)基材料为主。但是, PZT 基陶瓷的主 要生产原料为 $\mathrm{PbO}$, 它具有较大的毒性, 在生产和回 收处理过程中都会对人类健康和环境造成很大的损 害。因此, 研究性能优异的无铅压电陶瓷来替代铅基 压电陶瓷是目前压电陶瓷研究的紧迫任务。近年来, 无铅压电陶瓷的研究主要集中在碱金属铌酸盐和钛 酸铋盐等体系中。在这些体系中, 铌酸钾钠 $\left(\left(\mathrm{K}_{0.5} \mathrm{Na}_{0.5}\right) \mathrm{NbO}_{3}\right.$, 缩写为 $\left.\mathrm{KNN}\right)$ 基复合压电陶瓷由于 具有高的压电常数 $\left(d_{33}>200 \mathrm{pC} / \mathrm{N}\right)$ 、机电耦合系数 ( $\left.k_{\mathrm{p}} \sim 45 \%\right)$ 和居里温度 $\left(T_{\mathrm{c}}>400^{\circ} \mathrm{C}\right)$, 引起了人们的广泛 关注, 并有可能成为铅基压电陶瓷的替代品 ${ }^{[1-3]}$ 。但是, $\mathrm{KNN}$ 基陶瓷在制备过程中存在一个难题: 传统工艺 合成的粉体颗粒度大(通常在微米级), 团聚严重, 因 而烧结活性差，而高温下烧结造成 $\mathrm{K}$ 和 $\mathrm{Na}$ 等元素的 大量挥发, 很难得到致密的陶瓷烧结体, 严重影响 了该类材料电学性能的提升及在器件上的应用 ${ }^{[4-5]}$ 。 采用超细粉体, 特别是纳米粉体是提高粉体烧结活 性的一种有效方法 ${ }^{[6-7]}$, 该方法能显著降低陶瓷的 烧结温度, 减少钾钠的挥发, 从而有利于制备高致 密性陶瓷。

另一方面, 压电器件结构的微型化和集成化, 要 求介质层中的晶粒尺寸降到亚微米级甚至纳米级 ${ }^{[8]}$ 。 而由于纳米铁电陶瓷具有独特的晶界和电畴结构, 并具有 “临界尺寸”, 即当尺寸降低到某一值时, 其 铁电性能将会消失, 因此纳米铁电陶瓷的结构与铁 电性能的变化引起了广泛关注, 成为压电铁电材料 领域新的研究热点, 但是目前纳米铁电陶瓷的研究 主要局限于钛酸钡陶瓷体系 ${ }^{[9-11]}$ 。

因此, 采用纳米粉体制备纳米晶 $\mathrm{KNN}$ 陶瓷有 望在较低温度下获得高致密性陶瓷体, 并有可能表 现出独特的结构与电性能。但是相关研究却鲜有报 道, 这主要是由于纳米 $\mathrm{KNN}$ 粉体制备以及纳米陶 瓷烧结困难。虽然李月明 ${ }^{[7]}$ 和 $\mathrm{Liu}^{[12]}$ 等均采用水热 方法合成了 $\mathrm{KNN}$ 体系粉体, 但是获得的粉体不仅 颗粒尺度仍在微米级(甚至高于固相法合成粉体粒 度), 并且由于水热极限温度的限制, 粉体在自密闭 高压条件下呈两分相结晶, 难以满足后续纳米陶瓷 的制备需求。

本课题组前期工作开发了一种新型溶胶-凝胶 法, 可在较低温度下制备纳米 $\mathrm{KNN}$ 粉体, 制备出的 粉体直径只有 $30 \mathrm{~nm}$, 且分散良好, 无团聚现象 ${ }^{[13]}$ 。 本工作以该新型溶胶一凝胶法制备的平均晶粒尺寸 为 $30 \mathrm{~nm}$ 的 $\mathrm{KNN}$ 粉体为原料, 采用放电等离子体烧 结工艺 (Spark Plasma Sintering, SPS), 制备纳米
KNN 陶瓷, 并对该陶瓷的相结构、微观形貌、介电 性能和铁电性能进行研究。

\section{1 实验方法}

实验采用的原料包括: 铌的前驱物溶液 ${ }^{[13]}$, 碳 酸钠 $\left(\mathrm{Na}_{2} \mathrm{CO}_{3}, 99 \%\right)$, 碳酸钾 $\left(\mathrm{K}_{2} \mathrm{CO}_{3}, 99 \%\right)$, 氨水 $\left(\mathrm{NH}_{3} \cdot \mathrm{H}_{2} \mathrm{O}, 25.0 \sim 28.0 \%\right)$, 乙酸 $\left(\mathrm{CH}_{3} \mathrm{COOH}, 99.5 \%\right)$ 和 柠檬酸 $\left(\mathrm{C}_{6} \mathrm{H}_{8} \mathrm{O}_{7} \cdot \mathrm{H}_{2} \mathrm{O}, 99.5 \%\right)$ 。以上试剂使用前未经 进一步的纯化。

实验流程如下: 采用新型溶胶一凝胶法 ${ }^{[13]}$, 在 升温速率 $20{ }^{\circ} \mathrm{C} / \mathrm{min}$, 陆烧温度 $500^{\circ} \mathrm{C}$, 㷽烧时间 $5 \mathrm{~h}$ 的条件下制备 $\mathrm{KNN}$ 纳米粉体。然后将制备的 $3 \mathrm{~g}$ $\mathrm{KNN}$ 粉体装入 $\phi 20 \mathrm{~mm}$ 的石墨模具并手动预压, 在 日本住友石炭矿业株式会社生产的 SPS-3.20 MK-V 放电等离子烧结机上进行烧结。烧结温度为 $900^{\circ} \mathrm{C}$, 烧结压力为 $30 \mathrm{MPa}$, 升温速率为 $120^{\circ} \mathrm{C} / \mathrm{min}$ 左右, 真空度为 $8 \mathrm{~Pa}$, 烧结时间为 $1 \mathrm{~min}$ 。烧结体呈现黑色, 主要是由于模具渗碳和真空烧结引起的高浓度氧缺 陷所致。烧结体置于 $800^{\circ} \mathrm{C}$ 氧气中退火 $2 \mathrm{~h}$, 以去除 陶瓷体中的碳残留和消除氧缺陷, 退火后的陶瓷体 呈现乳白色。

为了对比, 采用传统固相法(Conventional Solid Sintering, CSS), 在 $1110^{\circ} \mathrm{C} 、 2 \mathrm{~h}$ 的烧结条件下制备 普通 KNN 陶瓷。

陶瓷样品经切割、表面研磨、抛光至厚度为 $1 \mathrm{~mm}$ 后可进行密度、相结构及形貌观察。将银浆涂 敷在样品上下表面, 在 $700^{\circ} \mathrm{C}$ 烧渗银电极后可进行 电学性能的测量。

采用 METTLER XS104 密度测试天平基于阿 基米德排水法测定陶瓷密度。通过 JEOL-JEM 2000 型透射电子显微镜(TEM)观测所得的明场形貌和衍 射花样, 确定所制备的 KNN 纳米粉体的形貌、晶粒 尺寸和结构。采用 Hitachi S-3500 型扫描电子显微 镜观察陶瓷断面经抛光腐蚀后的微观形貌。XRD 测 试采用 Bruker Diffractometer (AXS D8 ADVANCE) 型 $\mathrm{X}$ 射线衍射仪, 软件包括基本数据处理软件 EVA 以及峰形模拟和基本参数拟合软件 TOPAS 2P, 装备有 Gobel 镜, 铜靶 $(\lambda=0.15406 \mathrm{~nm}), 40 \mathrm{kV}$, $40 \mathrm{~mA}, \theta-2 \theta$ 模式, 扫描步长 $0.02 \%$ step, 扫描速率 $6 \%$ min。采用 Agilent E4980A 宽频 LCR 数字电桥测 量陶瓷样品的电容值 $C$ 和介电损耗 $\tan \delta$, 并计算介 电常数, 利用相对介电常数随温度的变化情况来确 定样品的相变温度及介电特性。电滞回线通过由计 算机控制的 Sawyer-Tower 电路自动测试系统完成, 
所用设备为 AixACCT-TF2000 型铁电参数测试仪。

\section{2 结果与讨论}

图 1 为采用新型溶胶一凝胶法制备的 $\mathrm{KNN}$ 纳米 粉体的透射电镜及电子衍射照片。可以看出, 粉体 形貌接近正方形，团聚小且大小比较均一，平均晶 粒尺寸在 $30 \mathrm{~nm}$ 左右。从电子衍射图案上可以看出, KNN 纳米粉体为单相结构，其衍射斑点符合正交对 称结构, 其中图中标出的三个衍射斑点分别对应于 (001)、(111)和(100)晶面。

对以纳米粉体为原料，经 SPS 制备的 KNN 陶瓷 和采用 CSS 制备的 KNN 陶瓷，分别进行密度测试，

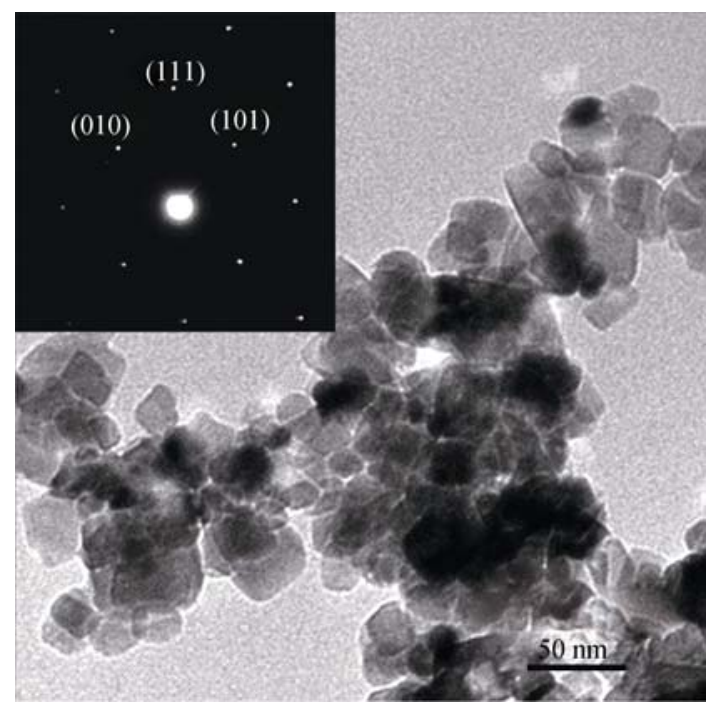

图 $1 \mathrm{KNN}$ 纳米粉体的透射电镜及电子衍射照片

Fig. 1 TEM image and SAED pattern of KNN nano powders

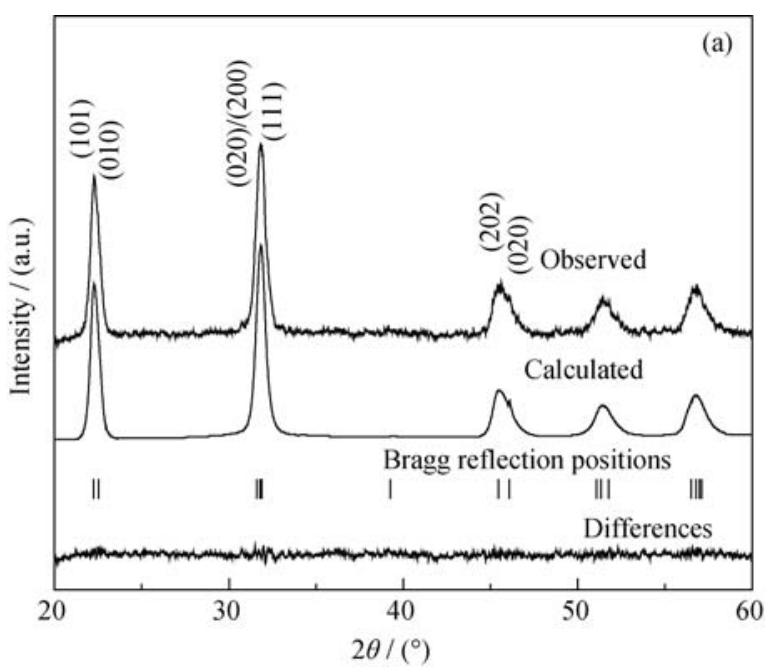

结果显示采用 CSS 制备的 KNN 陶瓷相对密度仅为 $90.4 \%$ ，与文献中的报道一致 ${ }^{[3,14-15]}$ 。这主要是由于 传统工艺制备的微米粉体团聚严重, 烧结活性差以 及 $\mathrm{Na}$ 和 $\mathrm{K}$ 等元素在高温下极易挥发, 很难得到致密 的陶瓷体。与之相比, 经 SPS 制备的 KNN 陶瓷相对 密度高达 99.3\%, 远大于采用 CSS 制备的 KNN 陶瓷。 图 2 为分别采用 SPS(a)和 CSS(b)制备样品的 SEM 照 片。由图 2 可以看出, 以纳米粉体为原料, 经 SPS 制 备的 KNN 陶瓷其晶粒尺寸在 $40 \mathrm{~nm}$ 左右, 并且其晶 粒大小比较均匀, 基本无气孔存在。而采用 CSS 制备 的 KNN 陶瓷，晶粒尺寸约为 $5 \mu \mathrm{m}$ 左右，并且晶粒大 小不均匀，存在明显的气孔。这说明实验采用的纳米 粉体具有很高的烧结活性，而采用 SPS 技术有效地利 用了通过电能产生的焦耳热、加压造成的塑性变形以 及粉体间放电所产生的自发热作用，有效地降低了纳 米粉的烧结温度，并在非常短的时间内获得高致密性 的纳米晶 KNN 陶瓷。

图 3 为采用 $\operatorname{SPS}(a)$ 和 $\operatorname{CSS}(b)$ 制备样品的 XRD
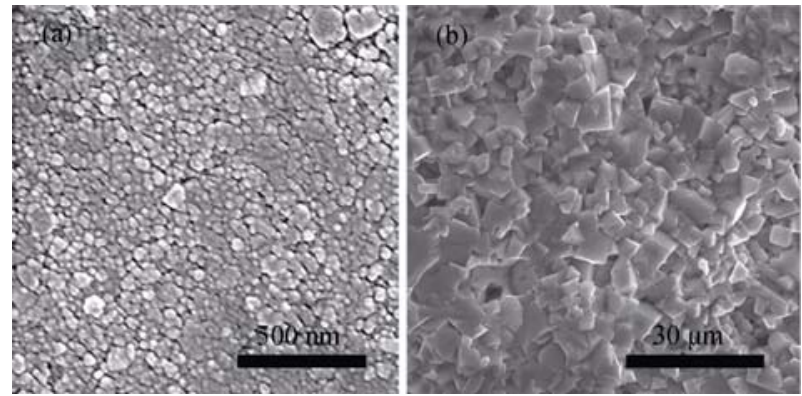

图 2 分别采用 SPS (a)和 CSS(b)制备样品的 SEM 照片 Fig. 2 SEM images of KNN samples sintered by SPS (a) and CSS (b) methods

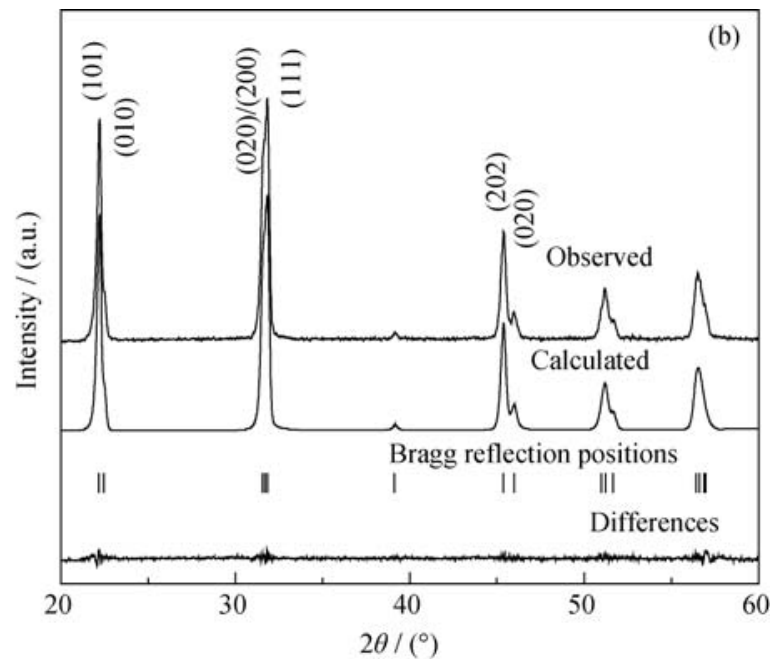

图 3 采用 SPS (a)和 CSS(b)制备样品的 XRD 图谱及 Rietveld 全谱精修结果

Fig. 3 XRD patterns and Rietveld refinement profile of KNN samples sintered by SPS (a) and CSS (b) methods 
图谱及 Rietveld 全谱精修结果, 可以看出, 采用 SPS 制备得到的陶瓷为纯钙钛矿相结构, 并且衍射峰发 生了明显的宽化, 由谢乐公式可以计算得到陶瓷的 晶粒尺寸为 $36.1 \mathrm{~nm}$, 这与 SEM 的测试结果比较一 致。其相结构为正交相, 晶格常数为: $a=$ $0.5611(8) \mathrm{nm}, b=0.3930(5) \mathrm{nm}, c=0.5648(0) \mathrm{nm}$, 误 差因子 $R_{\mathrm{wp}}$ 为 $9.91 \%$ 。与之相比, 采用 CSS 制备得 到的陶瓷仍为纯钙钛矿相结构, 但是衍射峰明显变 得尖锐, 说明其晶粒显著长大。其相结构同样为正 交相, 晶格常数为: $a=0.5627(0) \mathrm{nm}, b=0.3937(9) \mathrm{nm}$, $c=0.5654(3) \mathrm{nm}$ ，误差因子 $R_{\mathrm{wp}}$ 为 $8.56 \%$ 。

图 4 为采用不同方法制备的 KNN 陶瓷 $10 \mathrm{kHz}$ 下介电常数和损耗的温度谱。KNN 陶瓷室温以上存 在两个相变, 分别为 $200^{\circ} \mathrm{C}$ 左右的正交-四方相变和 $410^{\circ} \mathrm{C}$ 左右的四方-立方相变。由图 4 可以看出; 不 同方法制备的 KNN 陶瓷居里温度并没有明显变化, 但是 SPS 制备得到的纳米晶 KNN 陶瓷其介电常数 随温度变化不明显。CSS 制备的微米晶陶瓷室温下 的介电常数为 150 , 居里温度处的介电常数为 1435 , 比室温下的介电常数增加了 $856 \%$; 而 SPS 制备的 纳米晶 $\mathrm{KNN}$ 陶瓷室温下的介电常数为 341 , 居里温 度处的介电常数为 894 , 仅比室温下的介电常数增 加了 $162 \%$ 。这说明 SPS 制备的纳米晶 KNN 陶瓷的 介电常数在测试温度区间内较为平坦, 更有利于开 发高温度稳定性的多层陶瓷电容器材料。按照两相 系统串并联混合模型, 假设陶瓷体的介电常数由晶 粒介电常数和晶界介电常数两部分的贡献组成, 并 满足下列关系式 ${ }^{[16]}$ :

$$
\frac{D_{\mathrm{s}}}{\varepsilon_{\mathrm{r}, \mathrm{s}}}=\frac{D_{\mathrm{g}}}{\varepsilon_{\mathrm{r}, \mathrm{g}}}+\frac{D_{\mathrm{gb}}}{\varepsilon_{\mathrm{r}, \mathrm{gb}}}
$$

式中

$\varepsilon_{\mathrm{r}}$ 一陶瓷体的介电常数;

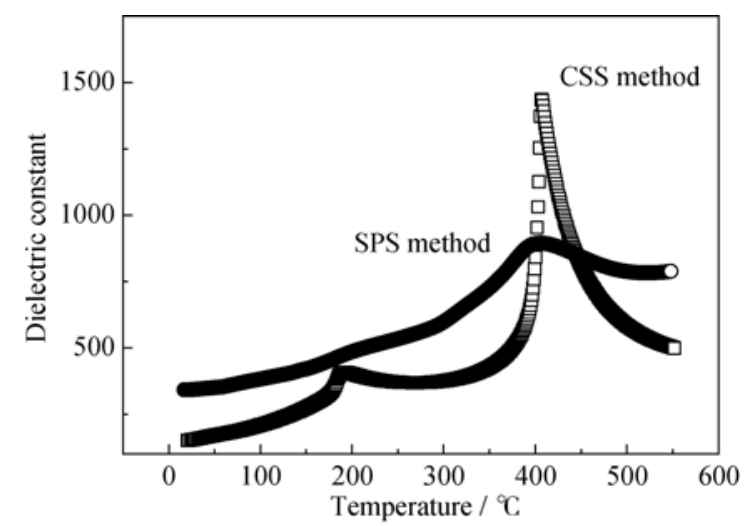

图 4 不同方法制备的 KNN 陶瓷介电常数温度谱

Fig. 4 Temperature dependence of dielectric constant of KNN ceramics sintered by different methods $\varepsilon_{\mathrm{r}, \mathrm{g}}$ 一晶粒的介电常数;

$\varepsilon_{\mathrm{r}, \mathrm{gb}}$ 一晶界的介电常数;

$D_{\mathrm{g}}$ 一晶粒的厚度;

$D_{\mathrm{gb}}$ 一晶界区的厚度;

$D_{\mathrm{s}}$ 一陶瓷体的等效厚度, $D_{\mathrm{s}}=D_{\mathrm{g}}+D_{\mathrm{gb}}$ 。

晶粒一般为铁电相, 其介电常数 $\varepsilon_{\mathrm{r}, \mathrm{g}}$ 比较大, 且在相变处变化明显; 而晶界通常由非铁电的玻 璃相组成, 其介电常数 $\varepsilon_{\mathrm{r}, \mathrm{gb}}$ 较低, 可认为是一常数, 且随温度变化不明显。本实验中, 晶粒尺寸引起的 KNN 陶瓷介电常数的变化是由两方面原因造成的: 一方面, 随着晶粒尺寸的降低, 晶界相在陶瓷体中 的比例逐渐增加, 特别是当陶瓷晶粒尺寸降低到 $100 \mathrm{~nm}$ 以下时，晶界相的比例明显增加，使得 $D_{\mathrm{g}}$ 减小, 而 $D_{\mathrm{gb}}$ 增加, 引起了陶瓷体介电常数的下降; 另一方面，随着 KNN 陶瓷晶粒尺寸的降低，晶界 相对电畴转向的抑制作用增强，这就造成晶粒介 电常数及其温度响应程度的降低。这两方面的综合 作用就会造成 SPS 制备的纳米晶 KNN 陶瓷介电常 数在整个测试温度区间内较为平坦, 并且低于相 对密度相同的微米晶 KNN 陶瓷。但是, 采用 CSS 制备的 KNN 陶瓷相对密度仅为 $90.4 \%$, 大量气孔 的存在对陶瓷体的介电常数产生了严重影响, 因 此, 采用 SPS 制备的纳米晶 KNN 陶瓷介电常数在 大部分温度区间内反而高于 CSS 制备的普通 KNN 陶瓷。

由图 4 还可以看出：与 CSS 制备的微米晶陶瓷 居里温度处介电常数显示为一个尖锐的峰不同, SPS 制备的纳米晶 KNN 陶瓷显示为一段较为圆滑 的弧线, 这说明纳米晶 KNN 陶瓷有明显的弥散相 变现象。对于弛豫铁电体, 在居里温度附近服从 Uchino-Nomura 方程 ${ }^{[17]}$ :

$$
\frac{1}{\varepsilon_{\mathrm{r}}}-\frac{1}{\varepsilon_{\mathrm{r}, \max }}=\frac{\left(T-T_{\max }\right)^{\gamma}}{C}
$$

式中

$\varepsilon_{\mathrm{r}, \max }$ 一最大介电常数;

$T_{\text {max }}$ 一最大介电常数处 $\varepsilon_{\mathrm{r}, \text { max }}$ 对应的温度;

$C$ 一居里型常数;

$\gamma$ 一描述相变弥散程度的因子, $\gamma$ 值为 1 时, 陶瓷 体系为正常铁电体; $\gamma$ 值为 2 时，陶瓷体系为完全的 弛豫铁电体。

为了进一步证实不同晶粒尺寸 $\mathrm{KNN}$ 陶瓷陶瓷 弛豫性能, 以 $\ln \left(1 / \varepsilon_{\mathrm{r}}-1 / \varepsilon_{\mathrm{r}, \max }\right)$ 对 $\ln \left(T-T_{\max }\right)$ 作图, 直线 的斜率即为表征相变弥散程度的因子 $\gamma$, 实验结果和 拟合结果如图 5 所示。拟合结果表明, CSS 制备的微 米晶 $\mathrm{KNN}$ 陶瓷, 其 $\gamma$ 值为 1.00 , 说明其为正常铁电 


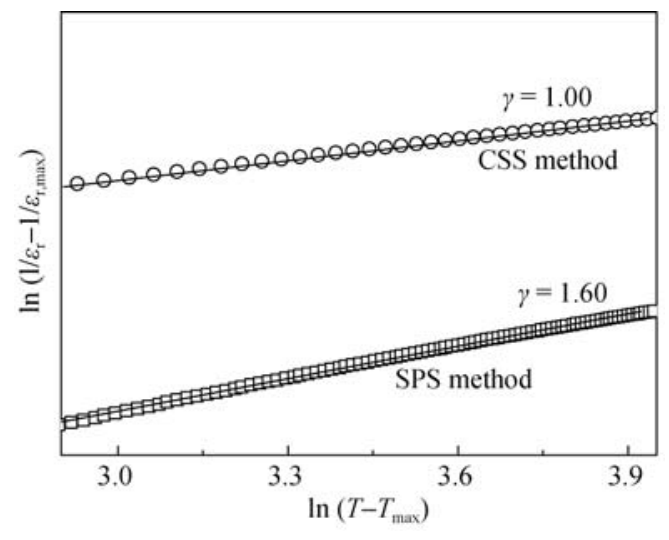

图 5 不同方法制备的 $\mathrm{KNN}$ 陶瓷 $\ln \left(1 / \varepsilon_{\mathrm{r}}-1 / \varepsilon_{\mathrm{r}, \max }\right)$ 随 $\ln \left(T-T_{\max }\right)$ 的变化及拟合结果

Fig. $5 \ln \left(1 / \varepsilon_{\mathrm{r}}-1 / \varepsilon_{\mathrm{r}, \max }\right)$ as a function of $\ln \left(T-T_{\max }\right)$ and fitting lines for KNN ceramics sintered by different methods

体; 而 SPS 制备的纳米晶 KNN 陶瓷，其 $\gamma$ 值为 1.60 , 表现出明显的弛豫铁电体特征。在正常铁电体中由晶 粒尺寸导致的弥散相变现象有许多报道, 弥散相变可 用在细晶陶瓷中存在的晶粒尺寸波动来解释 ${ }^{[18-19]}$, 当 晶粒尺寸降低到纳米级别时, 居里温度对晶粒尺寸 的变化敏感程度明显增加, 即晶粒尺寸的微小变化 也会引起居里温度的不同, 纳米陶瓷体中的晶粒尺 寸的分布是有一定范围的，因此纳米晶 KNN 陶瓷 具有明显的弛豫铁电体特征。

图 6 为采用不同方法制备的 KNN 陶瓷的电滞 回线，由图 6 可以看出，不同晶粒尺寸的 $\mathrm{KNN}$ 陶瓷 电滞回线存在明显差别, 其铁电参数分别为: CSS 制 备的微米晶 $\mathrm{KNN}$ 陶瓷, 矫顽场为 $13.4 \mathrm{kV} / \mathrm{cm}$, 剩余极 化为 $15.3 \mu \mathrm{C} / \mathrm{cm}^{2}$, 这也与文献报道的结果相近 ${ }^{[15]}$ 。而 SPS 制备的纳米晶 KNN 陶瓷，矫顽场为 $13.5 \mathrm{kV} / \mathrm{cm}$, 剩余极化为 $1.5 \mu \mathrm{C} / \mathrm{cm}^{2}$ 。与传统微米晶陶瓷相比, 纳 米晶 KNN 陶瓷矫顽场变化并不明显, 但是剩余

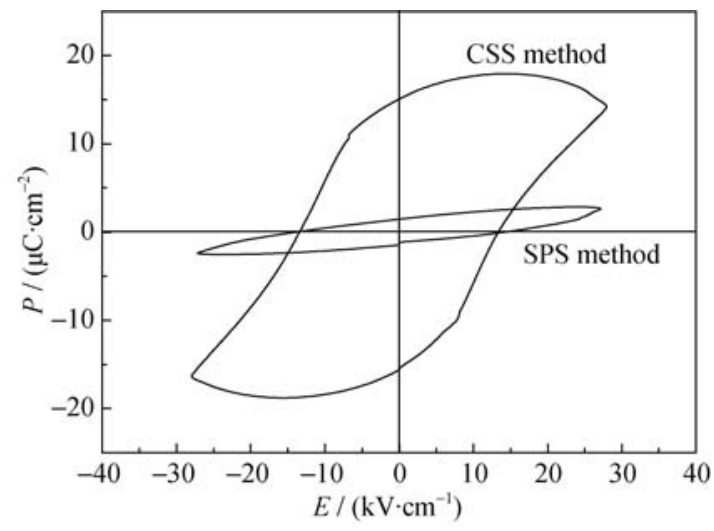

图 6 不同方法制备的 $\mathrm{KNN}$ 陶瓷的电滞回线

Fig. 6 Ferroelectric hysteresis loop of KNN ceramics sintered by different methods
极化仅为微米晶陶瓷的 $10 \%$ 。该现象与放电等离子 烧结 $\mathrm{BiFeO}_{3}$ 陶瓷的结果比较类似 ${ }^{[20]}$ 。晶粒尺寸的减 小, 一方面晶界相的作用增强, 铁电畴的尺寸降低, 不仅导致晶粒的自发极化强度的减小，而且增加了 经历反向畴生长过程的晶粒数量 ${ }^{[21]}$; 另一方面引起 了晶界(非铁电相)在陶瓷体中所占比例的增加, 所 以晶粒尺寸的减小造成 KNN 陶瓷剩余极化强度的 降低。由图 6 也可看出, 虽然纳米晶 KNN 陶瓷的铁 电性能发生了明显的弱化, 但是仍具有明显的电滞 回线现象，也就是说，如果 KNN 陶瓷具有 “临界尺 寸”，那么其值应该在 $40 \mathrm{~nm}$ 以下。

\section{3 结论}

以新型溶胶-凝胶法制备的平均晶粒尺寸为 $30 \mathrm{~nm}$ 的 $\mathrm{KNN}$ 粉体为原料, 采用 SPS 工艺, 在烧结 温度为 $900^{\circ} \mathrm{C}$, 烧结压力 $30 \mathrm{MPa}$, 保温时间 $1 \mathrm{~min}$ 的烧结条件下，制备得到纯正交相，相对密度高达 $99.3 \%$ ，平均晶粒尺寸为 $40 \mathrm{~nm}$ 的纳米 $\mathrm{KNN}$ 陶瓷， 并对该陶瓷的相结构、微观形貌、介电性能、铁电 性能进行了研究。结果表明, 与 CSS 制备的普通微 米晶陶瓷不同, 纳米 KNN 陶瓷表现出独特的电学 性能, 其介电常数为 341 , 并且随温度变化不明显, 表现出明显的介电弛豫现象, 弥散因子 $\gamma$ 为 1.60 , 并 具有明显的电滞回线现象，矫顽场为 $13.5 \mathrm{kV} / \mathrm{cm}$, 剩余极化为 $1.5 \mu \mathrm{C} / \mathrm{cm}^{2}$ 。如果 $\mathrm{KNN}$ 陶瓷具有 “临界 尺寸”，那么其值应该在 $40 \mathrm{~nm}$ 以下。

\section{参考文献:}

[1] SAITO Y, TAKAO H, TANI T, et al. Lead-free piezoceramics. Nature, 2004, 432(4): 84-87.

[2] GUO Y P, KAKIMOTO K, OHSATO H.Phase transitional behavior and piezoelectric properties of $\mathrm{Na}_{0.5} \mathrm{~K}_{0 .} \mathrm{NbO}_{3}-\mathrm{LiNbO}_{3}$ ceramics.Applied Physics Letters, 2004, 85(18): 4121-4123.

[3] KLEIN N, HOLLENSTEIN E, DAMJANOVIC D, et al. A study of the phase diagram of $(\mathrm{K}, \mathrm{Na}, \mathrm{Li}) \mathrm{NbO}_{3}$ determined by dielectric and piezoelec-tric measurements, and raman spectroscopy. Journal of Applied Physics, 2007, 102(1): 014112-1-8.

[4] MAEDER M D, DAMJANOVIC D, SETTER N. Lead free piezoelectric materials. Journal of Electroceramics, 2004, 13(2): 385-392.

[5] CHU S Y, WATER W, JUANG Y D, et al. Properties of ( $\mathrm{Na}, \mathrm{K}^{2} \mathrm{NbO}_{3}$ and ( $\mathrm{Li}, \mathrm{Na}, \mathrm{K} \mathrm{NbO}_{3}$ ceramic mixed systems. Ferroelectrics, 2003, 287(1): 23-33.

[6] HOU Y D, ZHU M K, HOU L, et al. Synthesis and characterization of lead-free $\mathrm{K}_{0.5} \mathrm{Bi}_{0.5} \mathrm{TiO}_{3}$ ferroelectrics by Sol-Gel technique. 
Journal of Crystal Growth, 2005, 273(3/4): 500-503.

[7] LI YUE-MING, LIU HU, SHEN ZONG-YANG, et al. Preparation of $(\mathrm{K}, \mathrm{Na}) \mathrm{NbO}_{3}$-based lead-free piezoelectric ceramic by microwave- hydrothermal method. Journal of the Chinese Ceramic Society, 2011, 39(12): 1922-1927.

[8] KISHI H, MIZUNO Y, CHAZONO H. Base-metal electrodemultilayer ceramic capacitors: Past, present and future perspectives. Japanese Journal of Applied Physics, 2003, 42(1): 1-15.

[9] BUSCAGLiA M T, BUSCAGLiA V, VIVIANi M, et al. Ferroelectric properties of dense nanocrystalline $\mathrm{BaTiO}_{3}$ ceramics. Nanotechnology, 2004, 15(9): 1113-1117.

[10] SAAD M M, BAXTER P, BOWMAN R M, et al. Intrinsic dielectric response in ferroelectric nano-capacitors. Journal of Physics: Condensed Matter, 2004, 16(41): L451-L456.

[11] MITOSERIU L, HARNAGEA C, NANNI P, et al. Local switching properties of dense nanocrystalline $\mathrm{BaTiO}_{3}$ ceramics. Applied Physics Letters, 2004, 84(13): 2418-2420.

[12] LIU N, WANG K, LI J F, et al. Hydrothermal synthesis and spark plasma sintering of $(\mathrm{K}, \mathrm{Na}) \mathrm{NbO}_{3}$ lead-free piezoceramics. Journal of the American Ceramic Society, 2009, 92(8): 1884-1887.

[13] WANG C, HOU Y D, GE H Y, et al. Sol-Gel synthesis and characterization of lead-free LNKN nanocrystalline powder. Journal of Crystal Growth, 2008, 310(22): 4635-4639.

[14] SHEN Z Y, WANG K, LI J F. Combined effects of Li content and sintering temperature on polymorphic phase boundary and electri- cal properties of $\mathrm{Li} / \mathrm{Ta}$ co-doped $(\mathrm{Na}, \mathrm{K}) \mathrm{NbO}_{3}$ lead-free piezoceramics. Applied Physics A, 2009, 97(4): 911-917.

[15] ZHANG LI-MIN, ZHANG BO-PING, LI JING-FENG, et al. Normal sintering of lead-free piezoceramic potassium sodium niobate and its electrical properties. Journal of the Chinese Ceramic Society, 2007, 35(1): 1-5.

[16] WANG H C, SCHULZE W A. The role of excess magnesium oxide or lead oxide in determining the macrostructure and properties of lead magnesium niobate. Journal of the American Ceramic Society, 1990, 73(4): 825-832.

[17] UCHINO K, NOMURA S. Critical exponents of the dielectric constants in diffused-phase-transition crystals. Ferroelectrics, 1982, 44(1): 55-61.

[18] CHATTOPADHYAY S, AYYUB P, PALKAR V R, et al. Size-induced diffuse phase-transition in the nanocrystalline ferroelectric $\mathrm{PbTiO}_{3}$. Physical Review B, 1995, 52(8): 13177-13183.

[19] PARKY V, LEE W J, KIM H G. Particle-size-induced diffuse phase transition in the fine-particle barium titanate porcelains. Journal of Physics: Condensed Matter, 1997, 9(43): 9445-9456.

[20] MAZUMDER R, CHAKRAVARTY D, Bhattacharya D, et al. Spark plasma sintering of $\mathrm{BiFeO}_{3}$. Materials Research Bulletin, 2009, 44(3): 555-559.

[21] KANG AI-GUO, DENG XIANG-YUN, WANG XIAO-HUI, et al. The dimension effects on hysteresis loops of fine grain $\mathrm{BaTiO}_{3}$ ceramics. Journal of Functional Materials, 2005, 36(11): 1712-1714. 